\title{
Factores que condicionan el rendimiento académico de los alumnos recursantes de Biofísica en la FOUNT.
}

Factors that restudents of Biophysics determine the academic performance

of the pupils in the FOUNT.

Fecha de Recepción 23 de mayo de 2011
Factores que condicionan o rendimento académico dos alunos recursantes de Biofisica na FOUNT.

Aceptado para su publicación

8 de junio de 2011

\section{J. E. Córdoba}

Cátedra Biofisica. Facultad de Odontología San Miguel de Tucumán.

S. M. Merletti

Cátedra Biofisica. Facultad de Odontología

San Miguel de Tucumán.

M. S. Alderete

Cátedra Biofisica. Facultad de Odontología San Miguel de Tucumán.

L. I. Perez

Cátedra Biofisica. Facultad de Odontología San Miguel de Tucumán.

\section{Resumen}

La asignatura Biofísica se dicta en $1^{\circ}$ año de la carrera de Odontólogo de la UNT con un promedio aproximado de 300 alumnos por año. Más del $50 \%$ no logra regularizar la materia y los alumnos libres de cada año académico algunas veces deben recursar hasta tres o cuatro veces. El fracaso de este importante grupo de alumnos ha generado una creciente preocupación en los docentes y la necesidad de indagar los motivos que producen estos resultados indeseables. EI OBJETIVO de este trabajo es investigar la metodología de estudio, dedicación, actitud hacia la asignatura en alumnos recursantes con el fin de implementar estrategias que los motiven a lograr un aprendizaje significativo. METODO: se administró una encuesta a 80 recursantes, antes del $I^{\circ}$ examen parcial. Se indagó acerca de la metodología de estudio de la asignatura, consultas con docentes de la Cátedra, apoyo con profesores particulares, indicadores de comprensión de textos de cada tema, conocimientos previos, dedicación al estudio, interés por la materia, asistencia a clases teóricas, comprensión de enunciados de los parciales, comunicación con los docentes, entre otros. RESULTADOS: en el estudio de un tema, el $81 \%$ identifica los conceptos claves; el $60 \%$ los relacionan; el $62 \%$ los organizan jerárquicamente. Sólo el $20 \%$ consulta con docentes de la Cátedra y el $40 \%$ asiste a profesores particulares. El $50 \%$ hace un resumen del texto que estudia, pero solamente $8 \%$ realiza síntesis. El $59 \%$ manifiesta falta de conocimientos previos; el $47 \%$ expresa falta de dedicación e 
interés por la materia y sólo el $20 \%$ asiste a clases teóricas. En cuanto a la comprensión de enunciados de parciales, el $21 \%$ considera que son difíciles y el $22 \%$ que no hay buena comunicación con los docentes. CONCLUSIÓN: Se evidencia en los recursantes un abordaje superficial de los textos de la asignatura, poco aprovechamiento de las oportunidades de interaccionar académicamente con los docentes, poco interés, poca dedicación y conocimientos previos deficientes. Es necesario profundizar en la investigación de los factores que condicionan el rendimiento de los recursantes a fin de desarrollar estrategias personalizadas que les posibiliten involucrarse activamente en el proceso de aprendizaje.

\section{Palabras Clave}

Recursantes, desinterés, aprendizaje significativo

\section{Abstract}

Biophysics is dictated in First Year Dentist's career at UNT with an average of approximately 300 students per year. More than $50 \%$ does not manage to regularize the matter and free pupils out of each academic year should appeal until sometimes three or four times. The failure of this important students's group has generated an increasing worry in the teachers and the need to investigate the reasons that produce these undesirable results. The AIM of this study is to investigate methodology of study, dedication, attitude toward the subject in re - students in order to implement strategies that motivate them to achieve significant learning. METHOD: A survey was administered to 80 re-students before first test. They were inquired about the methodology of study of the subject, consult with teachers of Biophysics Department, support with personal tutors, indicators of understanding of texts, previous knowledge, dedication to the study, interest for the matter, assistance to theoretic classrooms, understanding of enunciates of the written tests, communication with the teachers, among other ones. RESULTS: In the study of a theme, the $81 \%$ say they identify key concepts, $60 \%$ they relate themselves; $62 \%$ they organize themselves according to the hierarchy. Only the $20 \%$ consults with Biophysics's teachers and the $40 \%$ helps personal tutors. The $50 \%$ makes out a summary of the text that it studies, but only $8 \%$ do synthesis. The $59 \%$ manifests lack of previous knowledge; $47 \%$ expresses lack of dedication and interest for the matter and only the $20 \%$ attends theoretic classrooms. As to the understanding of enunciates of tests, $21 \%$ says that they are difficult and $22 \%$ that does not have good communication with the teachers. CONCLUSION: A superficial boarding of the texts of the subject of study becomes evident in the re-students, little use of interaccionar's opportunities academically with the teachers, little interest, not much dedication and previous deficient knowledge. It is necessary to delve deeply into the investigation of the factors that condition the performance of the re-students in order to develop personalized strategies that they make them possible getting involved actively in the process of learning.

\section{Key Words}

Re-students, disinterestedness, significant learning.

\section{Resumem}

A matéria Biofísica dita-se em $I^{\circ}$ ano da carreira de Odontólogo da UNT com uma média aproximada de 300 alunos por ano. Mais de 50\% não consegue regularizar a matéria e os alunos livres da cada ano académico algumas vezes devem recursar até três ou quatro vezes. $O$ falhanço deste importante grupo de alunos gerou uma crescente preocupação nos docentes e a necessidade de indagar os motivos que produzem estes resultados indeseables. O OBJECTIVO deste trabalho é pesquisar a metodología de estudo, dedicación, atitude para a matéria em alunos recursantes com o fim de implementar estratégias que os motivem a conseguir uma aprendizagem significativa. METODO: administrou-se uma encuesta a 80 recursantes, dantes do $1^{\circ}$ exame parcial. Se indagó a respeito da metodología de estudo da matéria, consultas com docentes da Cátedra, apoio com professores particulares, indicadores de entendimento de textos da cada tema, conhecimentos prévios, dedicación ao estudo, interesse pela matéria, assistência a classes teóricas, entendimento de enunciados dos parciais, comunicação com os docentes, entre outros. RESULTADOS: no estudo de um tema, o $81 \%$ identifica os conceitos finques; o $60 \%$ relacionam-nos; o $62 \%$ organizam-nos hierarquicamente. Só o $20 \%$ consulta com docentes 
da Cátedra e o $40 \%$ assiste a professores particulares. O $50 \%$ faz um resumem do texto que estuda, mas somente $8 \%$ realiza síntese. O $59 \%$ manifesta falta de conhecimentos prévios; o $47 \%$ expressa falta de dedicación e interesse pela matéria e só o $20 \%$ assiste a classes teóricas. Em quanto ao entendimento de enunciados de parciais, o $21 \%$ considera que são difíceis e o $22 \%$ que não há boa comunicação com os docentes. CONCLUSÃO: Se evidência nos recursantes um abordaje superficial dos textos da matéria, pouco aprovechamiento das oportunidades de interaccionar academicamente com os docentes, pouco interesse, pouca dedicación e conhecimentos prévios deficientes. É necessário aprofundar na investigação dos factores que condicionan o rendimento dos recursantes a fim de desenvolver estratégias personalizadas que lhes possibilitem se envolver activamente no processo de aprendizagem.

\section{Palavras Finque}

Recursantes, desinterés, aprendizagem significativa.

\section{Introducción}

En el inicio del cursado de Biofísica los alumnos disponen del cronograma, bibliografía general y específica de cada unidad temática, condiciones de regularidad, fechas de trabajos prácticos y exámenes parciales con sus respectivas recuperaciones.

El propósito de brindar esta información es ubicar al estudiante en su rol de alumno de la carrera de Odontología para afianzar su sentimiento de pertenencia a un espacio socio-cultural como es la facultad.

Cabe destacar que los estudiantes conocen las condiciones de evaluación y requisitos del cursado. Pueden plantear cambios en las fechas de exámenes parciales, dentro de lo posible, por superposición con otras materias.

Según Alonso' Biofísica es una rama de la Biología que estudia problemas biológicos con métodos físicos. Explica los fenómenos de la vida en base al razonamiento con métodos derivados de la Física².

Los problemas que presentan los estudiantes en el aprendizaje de Física básica se producen en todos los niveles, inclusive en el ámbito universitario. Entonces, enseñar esta asignatura no es tarea sencilla, especialmente en carreras como Odontología, donde es una materia básica.

En general, el promedio de los últimos cinco años de alumnos que regularizan la materia es del $40 \%$. El porcentaje restante se encuentra en condición de alumnos libres.

Nescier, Althaus y Rocha $^{3}$ consideran preocupante el elevado número de estudiantes que incurre en una situación de fracaso, llevándolos a cursar en forma reiterada una asignatura.

Al respecto, Fornasero y Tumini ${ }^{4}$ definen a los recursantes como aquellos alumnos que reinciden en cursar nuevamente una materia. Estas autoras aseguran que esta situación produce frustración académica y lentitud en sus estudios.

En cuanto al perfil del recursante, Di Melfi, Escasany y Götz ${ }^{5}$ establecen que presenta bajo nivel de autoestima, alto grado de dificultad con la materia, amenaza de perder su grupo de estudio, superposición con materias del ciclo regular e inmadurez en su desarrollo pedagógico. Además afirman que es el mismo alumno que cuando se recupera del fracaso, más satisfacciones brinda al docente.

Los estudiantes que recursan una materia también muestran dificultad y demora para rendir los exámenes finales (Vera, $\left.{ }^{6}\right)$.

Además, como explican Fornasero y Benegas ${ }^{7}$ "la pedagogía actual no puede ignorar esta nueva condición de alumno, sino asumirla como otra realidad coexistente con la imagen del alumno de la modernidad".

Dice Edel Navarro ${ }^{8}$ que el fracaso académico tiene causas multifactoriales como habilidad social, autocontrol y expectativas del estudiante. En sus investigaciones encontró que el fracaso genera un sentimiento de humillación y falta de autovaloración.

También la Comisión de Políticas de Enseñanza de la Facultad de Ingeniería de la Universidad de la República9 afirma que el recursado pone en evidencia factores pertenecientes a tres niveles: el plano personal, como proyecto de vida; el plano institucional de la facultad y la universidad; y el plano nacional con sus problemáticas políticas, sociales y culturales, condicionantes del estudio universitario.

Según Ledesma y Ballestero ${ }^{10}$ los fracasos académicos en primer año de Psicología obedecen a factores como uso incorrecto del vocabulario propio de la disciplina, falta de estudio, limitación 
de comprensión, incoherencia en la expresión escrita.

Los aportes de Fornasero, Martella y Tumini" permiten establecer que el fracaso de este importante porcentaje de alumnos en el proceso de aprendizaje ha generado una creciente preocupación en los docentes e instaló la necesidad de indagar los motivos de estos resultados no deseados dentro de los estudios de grado.

Las investigaciones de Lozano, Díaz y Jorrat ${ }^{12}$ en entrevistas con alumnos recursantes demuestran que éstos reconocen como causa de su fracaso en el cursado la falta de dedicación y esfuerzo en el estudio. Además manifiestan el poco tiempo disponible para estudiar de libros de textos y su preferencia para estudiar de apuntes propios o ajenos. A esto se suma la escasa comprensión lectora que se evidencia en errónea interpre tación de preguntas escritas o situaciones pro blemáticas planteadas.

Otros investigadores como Monzón y Di Paolo ${ }^{13}$ indican que las competencias básicas durante un año de cursado para evitar el fracaso académico son: lectura comprensiva y rápida; expresión oral con desarrollo de un vocabulario científico y matemática básica con habilidades operacionales mínimas para desarrollar los conceptos físicos.

Los aportes de Blanca y col. ${ }^{14}$ en su trabajo Opinión de los alumnos recursantes sobre su trayectoria en la Universidad revelan que los estudiantes consideran que deben estudiar más, asistir a las clases teóricas y conocer las exigencias de las asignaturas.

En la Universidad no se puede estudiar de memoria ya que los nuevos conocimientos se deben integrar y relacionar con conocimientos previos. Por ello Golbach y col. ${ }^{15}$ estiman que es necesario investigar en los alumnos su metodología de estudio, las estrategias de aprendizaje y las motivaciones para aprender.

El informe de Fornasero, Martella y Tumini ${ }^{16}$ sobre la temática del alumno recursante muestra que la falta de estudios sistematizados es un escollo para el éxito universitario.

Medina ${ }^{17}$ agrega que para evitar el fracaso en la universidad es necesario desarrollar hábitos de estudio, asistir regularmente a clases teóricas con una presencia activa en las mismas.

En este sentido, con el fin de superar la metodología de enseñanza tradicional en las clases teóricas multitudinarias de Matemática I, Galindo y Cudmani ${ }^{18}$ recurren al uso de material instruccional. Así, evidencian modificaciones positivas en los resultados de los exámenes finales.

Advierten Lozano, Díaz y Jorrat ${ }^{12}$ que además de los hábitos de estudio es notorio el deterioro de la calidad y cantidad de los conocimientos previos sobre Física, Matemática y Lengua de los estudiantes en primer año universitario.

En un trabajo reciente Alderete, Merletti y Pérez ${ }^{19}$ sostienen que el bajo piso cognitivo de los estudiantes universitarios atentan contra el rendimiento académico y alarga la duración de las carreras.

Los estudios de Magnarelli y col. ${ }^{20}$ demuestran que la articulación de pequeños grupos con seminarios científicos, trabajos prácticos con laboratorio y la evaluación de pequeños grupos motivan a los alumnos y permiten la recuperación de conocimientos previos conduciendo a la integración conceptual.

D'Angelo y Palacio ${ }^{21}$ añaden a las técnicas anteriores, las actividades extra-áulicas: integración del estudiante al nuevo grupo social que favorece el crecimiento personal, el sentimiento de pertenencia a la carrera y el rendimiento académico.

El objetivo de este trabajo es investigar la metodología de estudio, dedicación, actitud hacia la asignatura en alumnos recursantes con el fin de implementar estrategias que los motiven a lograr un aprendizaje significativo.

\section{Método}

Se administró una encuesta a 80 (ochenta) alumnos recursantes antes del primer examen parcial. Se indagó acerca de la metodología de estudio de la asignatura, consultas con docentes de la Cátedra, apoyo con profesores particulares, indicadores de comprensión de textos de cada tema, conocimientos previos, dedicación al estudio, interés por la materia, asistencia a clases teóricas, comprensión de enunciados de los parciales, comunicación con los docentes, entre otros.

\section{Resultados}

En el estudio de un tema, el $81 \%$ identifica los conceptos claves; el $60 \%$ los relacionan; el $63 \%$ los organizan jerárquicamente. El $50 \%$ hace un resumen del texto que estudia, pero solamente 
$8 \%$ realiza síntesis. El $59 \%$ manifiesta falta de conocimientos previos; el $47 \%$ expresa falta de dedicación e interés por la materia y sólo el $20 \%$ asistieron a clase teórica.

En cuanto a la comprensión de enunciados de parciales, el $21 \%$ considera que son difíciles y el $23 \%$ que no hay buena comunicación con los docentes.

Cuando tuvieron dificultades con la materia, el $56 \%$ estudió con compañeros, el $20 \%$ realizó consultas con los docentes de la Cátedra, y el $40 \%$ asiste a profesores particulares.

Las tablas y gráficos siguientes muestran todos los resultados obtenidos.
Tabla I. Distribución de alumnos según su asistencia a clases teóricas.

\begin{tabular}{|l|c|c|}
\hline Item & $\mathrm{N}^{\circ}$ & $\%$ \\
\hline Siempre & 17 & 20 \\
\hline A veces & 49 & 58 \\
\hline Nunca & 20 & 32 \\
\hline
\end{tabular}

\section{Figura $\mathbf{I}$}

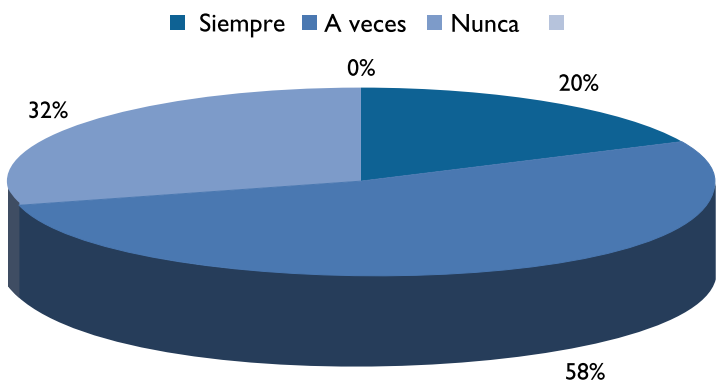

Figura 2.

Tabla 3. Distribución de alumnos según metodología de estudio.

\begin{tabular}{|l|c|c|}
\hline Item & $\mathrm{N}^{\circ}$ & $\%$ \\
\hline Leen varias veces & 38 & 48 \\
\hline Hacen resúmenes & 40 & 50 \\
\hline Marcan lo importante del texto & 42 & 53 \\
\hline
\end{tabular}

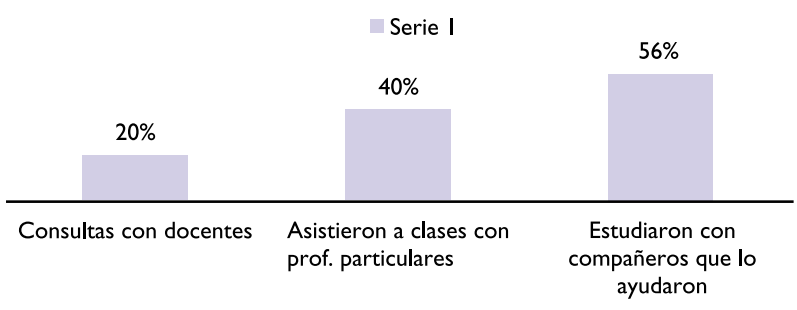

Figura 3.

\begin{tabular}{|c|c|}
\hline$N^{\circ}$ & $\%$ \\
\hline 16 & 20 \\
\hline 32 & 40 \\
\hline 45 & 56 \\
\hline
\end{tabular}

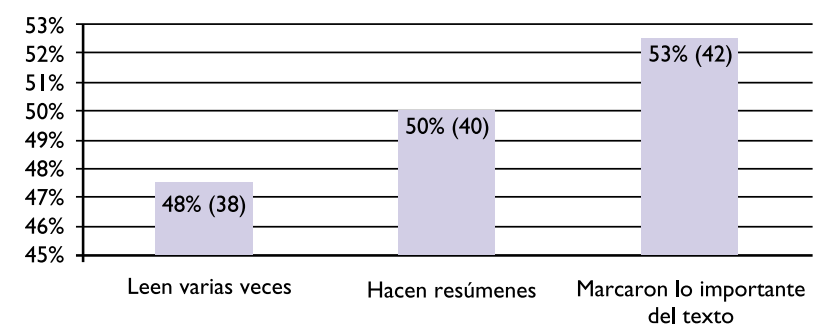


Tabla 4. Distribución de los alumnos según adquisición y comprensión de conceptos nuevos.

\begin{tabular}{|l|c|c|}
\hline Item & $\mathrm{N}^{\circ}$ & $\%$ \\
\hline $\begin{array}{l}\text { Relaciona los conceptos } \\
\text { nuevos con otros estudiados } \\
\text { anteriormente }\end{array}$ & 44 & 55 \\
\hline $\begin{array}{l}\text { Distingue cómo se } \\
\text { relacionan los conceptos }\end{array}$ & 48 & 60 \\
\hline $\begin{array}{l}\text { Organiza los conceptos por } \\
\text { orden de importancia }\end{array}$ & 50 & 63 \\
\hline \begin{tabular}{l} 
Identifica los conceptos claves \\
\hline
\end{tabular} & 65 & 81 \\
\hline
\end{tabular}

Tabla 5. Distribución de alumnos según dificultades en el cursado de Biofísica.

\begin{tabular}{|l|c|c|}
\hline Item & $N^{\circ}$ & $\%$ \\
\hline Modalidad de estudio & 38 & 48 \\
\hline Falta de dedicación al estudio & 38 & 48 \\
\hline $\begin{array}{l}\text { Falta de concentración } \\
\text { y esfuerzo }\end{array}$ & 39 & 49 \\
\hline \begin{tabular}{l} 
Falta de conocimientos previos \\
\hline
\end{tabular} & 47 & 59 \\
\hline
\end{tabular}

Tabla 6. Distribución de alumnos según dificultades en el cursado de Biofísica.

\begin{tabular}{|l|c|c|}
\hline Item & $\mathrm{N}^{\circ}$ & $\%$ \\
\hline $\begin{array}{l}\text { Parciales difíciles de } \\
\text { entender y resolver }\end{array}$ & 17 & 21 \\
\hline $\begin{array}{l}\text { Poca comunicación } \\
\text { con los docentes }\end{array}$ & 18 & 23 \\
\hline $\begin{array}{l}\text { Poca participación en los } \\
\text { trabajos prácticos }\end{array}$ & 22 & 28 \\
\hline
\end{tabular}

\section{Discusión}

Se ha estudiado, a través de esta investigación, la metodología de estudio, dedicación y actitud hacia la asignatura Biofísica en los alumnos recursantes de la FOUNT.
Figura 4.

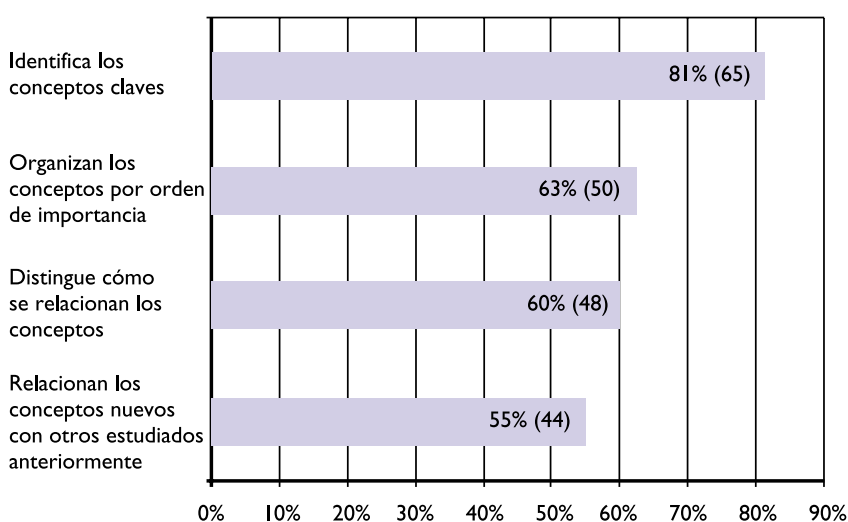

Figura 5.

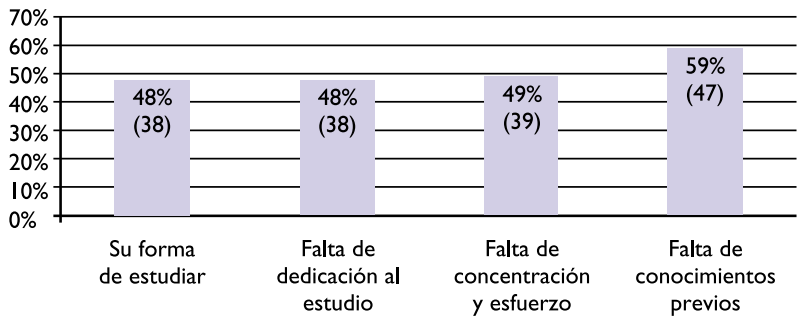

Figura 6.

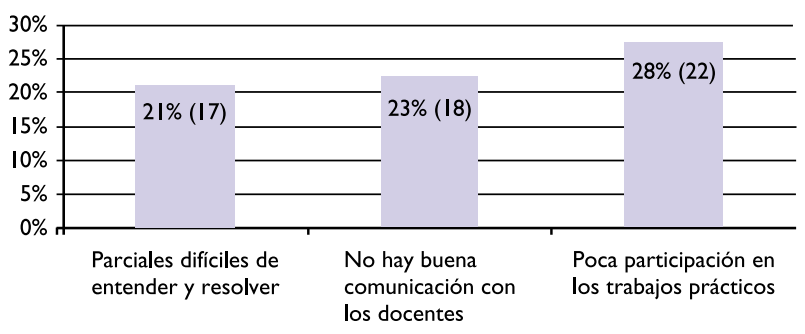

Se puede afirmar que algunas de las causas principales del fracaso académico son la falta de conocimientos previos, ausencia de metodologías de estudio y poca comunicación con los docentes. Sin embargo, Edel Navarro ${ }^{8}$ asegura que hay causas multifactoriales como falta 
de habilidad social, autocontrol y expectativas del estudiante.

Por otra parte, es probable que existan otros factores como los que considera la Comisión de Políticas de Enseñanza de la Facultad de Ingeniería de la Universidad de la República ${ }^{9}$ pertenecientes al plano personal, institucional y nacional.

Según los datos obtenidos, aproximadamente la mitad de los recursantes manifiestan falta de estudio, dedicación y esfuerzo. En este sentido, Ledesma y Ballesteros ${ }^{10}$ hacen notar que los fracasos académicos se deberían al uso incorrecto del vocabulario científico, falta de estudio e incoherencia en la expresión escrita.

Lozano, Diaz y Jorrat ${ }^{12}$ coinciden con la opinión de estos autores al afirmar que los estudiantes mencionan escasa comprensión lectora, lo que conduce a una mala interpretación de las consignas. Esto se evidencia en el bajo rendimiento en los exámenes parciales.

Podría preguntarse si existen otras causas que producen el bajo rendimiento en los alumnos recursantes y una respuesta sería la opinión de Monzón y Di Paolo ${ }^{13}$ quienes dicen que además de la falta de lectura comprensiva y rápida se observa falta de conocimientos de matemática básica, indispensable para comprender y aplicar temas de Física en situaciones problemáticas.

El bajo nivel de rendimiento encontrado también podría deberse a la falta de estudios sistematizados como sugieren Fornasero, Martella y Tumini". Sin embargo, según Alderete, Merletti y Pérez ${ }^{19}$, la causa del alargamiento de las carreras y el mal rendimiento académico serían el bajo piso cognitivo de los estudiantes universitarios.

Si los alumnos asistieran siempre a clases teóricas podrían organizar eficazmente los contenidos de la asignatura en cuanto a conceptos que por su complejidad necesitan la explicación personal, análisis de textos y ayuda en la interpretación de representaciones gráficas.

En concordancia, Blanca y col. ${ }^{14}$ y Medina ${ }^{17}$ mencionan que se debe asistir regularmente a clases teóricas. Esto fue comprobado en la presente investigación ya que menos de la cuarta parte de los encuestados asistieron a clases teóricas.

\section{Conclusión}

Se encontró que los alumnos recursantes muestran un abordaje superficial de los textos de la asignatura, poco aprovechamiento de las oportunidades de interaccionar académicamente con los docentes, poco interés, poca dedicación y conocimientos previos deficientes. También manifiestan mala comprensión de las consignas e incorrectas metodologías de estudio.

Se comprobó que un buen porcentaje estudia con compañeros que los ayudan y también asisten a profesores particulares sin concurrir a las clases teóricas de la asignatura.

De acuerdo a los resultados obtenidos, los autores del este trabajo consideran importante continuar con el estudio en dos direcciones: la implementación de estrategias motivadoras y la aplicación de un sistema tutorial. La asociación entre nuevas estrategias didácticas y las tutorías permitirían un mejor rendimiento de los alumnos recursantes.

De esta información surgen algunas propuestas:

- Motivar a los alumnos recursantes para asistir y aprovechar las clases teóricas de la materia, y consultar con los docentes de la Cátedra.

- Habilitar horarios de tutorías para orientar a estos alumnos con el objetivo de mejorar el rendimiento académico y su adaptación al ámbito universitario.

- Incorporar talleres a fin de facilitar las técnicas para la resolución de situaciones problemáticas tales como emisión de hipótesis, aplicación de fórmulas y análisis de los resultados.

Es necesario profundizar en la investigación de los factores que condicionan el rendimiento de los recursantes a fin de desarrollar estrategias personalizadas que les posibiliten involucrarse activamente en el proceso de aprendizaje. 


\section{Facultad de Odontología}

\section{ENCUESTA}

\section{Datos personales}

-Edad: años.

-Sexo $\square F \quad \square M$

-Tres últimos números del documento (DNI):

Su rendimiento en Física en la escuela secundaria fue:

$\square$ Muy bueno $\square$ Bueno $\square$ Regular $\square$ Deficiente

¿Le gusta Física?
$\square \mathrm{Si}$<smiles>[Nb]=C1CCC1</smiles>

¿Se encuentra cursando la asignatura "Biofisica" actualmente?

$\square$ Si

$\square$ No

I. ¿Cuántas veces recursó Biofisica anteriormente? veces

2. ¿Qué nota obtuvo en el primer parcial de Biofisica?
$\square$ Menos de 4
$\square$ de 4 a 6
$\square$ de 6 a 8
$\square$ de 8 a 10
No rendí

3. ¿Qué nota obtuvo en el segundo parcial?

$\square$ Menos de $4 \quad \square$ de 4 a $6 \quad \square$ de 6 a $8 \quad \square$ de 8 a $10 \quad \square$ No rendí

4. ¿Qué nota obtuvo en el tercer parcial?
$\square$ Menos de 4
$\square$ de 4 a 6
$\square$ de 6 a 8
$\square$ de 8 a 10
No rendí

5. ¿Asistía a clases teóricas?
$\square$ Siempre $\square$ A veces
$\square$ Nunca

6. ¿Asistía a clases de consulta en la Cátedra?
$\square$ Siempre $\square$ A veces $\square$ Nunca

7. En el estudio de un tema, usted utiliza:
$\square$ Libros de texto
$\square$ Guías de Cátedra
$\square$ Apuntes de clase teórica
Libros, guías y apuntes

8. Cuando tuvo dificultades en la asignatura:
$\square$ Asistió a clases con profesores particulares
$\square$ Recurrió a clases de consulta con los docentes de la Cátedra
$\square$ Estudió con compañeros que le ayudaron
$\square$ Otros. Indique: 


\section{Facultad de Odontología}

9. ¿Qué recurso utiliza para organizar los temas que estudia en la materia?

Marcar lo importante del texto

$\square$ Resúmenes

$\square$ Sintesis

$\square$ Fichas

Tablas

$\square$ Cuadros sinópticos
$\square$ Leer varias veces
$\square$ Esquemas

No conozco ningún recurso para organizar la información

10. En el estudio de cada tema, usted logra:

a)Identificar los conceptos claves $\square$ Si $\square$ No $\quad \square$ Pocas veces

b)Distinguir cómo se relacionan los conceptos $\square$ Si $\square$ No $\square$ Pocas veces

c) Organizar los conceptos por orden de importancia

$\square \mathrm{Si} \square$ No $\square$ Pocas veces

d) Relacionar los conceptos nuevos con otros estudiados anteriormente

$\square$ Si $\square$ No $\square$ Pocas veces

II. Marque cuáles fueron las causas de sus dificultades en el cursado de Biofisica. Indique con los números I, 2 ó 3 esas causas, asignando I a la mayor dificultad:

Falta de conocimientos previos

Su forma de estudiar

Falta de dedicación al estudio

Falta de concentración y esfuerzo

Dificultad para interpretar los textos

No pudo interpretar los enunciados de los parciales

Desinterés por el estudio de la Física

Ninguna

Otras. Indique:

12. Considera que los motivos de sus dificultades en Biofisica se deben a:

$\square$ Las clases teóricas tienen muchos alumnos

$\square$ Las clases prácticas tienen muchos alumnos

$\square$ Los exámenes parciales son dificiles de entender y resolver

$\square$ Los métodos de enseñanza utilizados no son adecuados

$\square$ No hay buena comunicación con los docentes

$\square$ La poca participación de los estudiantes en los Trabajos Prácticos

$\square$ Los contenidos desarrollados durante el tiempo de dictado son excesivos

Otras razones. Indique:

13. ¿Conoce los mapas conceptuales como estrategia de aprendizaje?

No conozco los mapas conceptuales

Conozco los mapas conceptuales pero no sé confeccionarlos

Sé confeccionar mapas conceptuales y los utilizo para estudiar 


\section{Referencias Bibliográficas}

I. Alonso, G. (2005). Biofísica. La ciencia y su enseñanza universitaria. Revista de la Facultad de Odontología (UBA). 20 (49): 21 - 26.

2. Alonso, G; Alippi, R. (1986). Biofísica. $3^{\mathrm{a}}$ Edición. Tekne: Buenos Aires: I.

3. Nescier I.; Althaus, R.; Rocha, A. (2007). Factores que condicionan la apropiación del conocimiento y su durabilidad en la asignatura Química en alumnos de Ingeniería Agronómica. Revista FAVE- Ciencias Agrarias 5/6 (I-2): 9l-96.

4. Fornasero, S.; Tumini, G. (2009). El alumno recursante: Prácticas innovadoras aplicadas al proceso de enseñanza y aprendizaje. III Congreso Internacional del Educación: 6 Versión on line: www.fhuc.unl.edu.ar/.../7\%20de\%20agosto\%20$\% 20$ Cronograma\%20de\%20Presentación\%20 de\%20Trabajos.pdf

5. Di Melfi,S.; Escasany, M.; Götz, E. (2005). Fracaso y recuperación del estudiante universitario. Universidad Católica Argentina. Versión on line: www.enduc.org.ar/comisfin/ponencia// 04-05. doc

6. Vera, P. ( ). Sistema de tutorías universitarias (ciclo básico).Universidad Tecnológica Nacional. Facultad regional Mendoza. Secretaría Académica. Dirección de orientación psicopedagógica: I- 17.

7. Fornasero, S.; Benegas, A. (2007). El alumno recursante como problemática de la pedagogía actual. I Jornadas Nacionales y I Latinoamericanas de Pedagogía Universitaria. Enseñar y aprender en la Universidad. "Culturas y educación en la Universidad: problemas y perspectivas". UNSAM. San Martín. Buenos Aires: 5. V. on line: ww.unsam.edu.ar/...pedagogiall/ProgramadetalladoComisiones.pdf
8. Edel Navarro, R. (2003). Factores asociados al Rendimiento Académico. Revista lberoamericana de Educación. México. ISSN:I68I-5653. Version on line: www.rieoei.org/investigacion/5 I2Edel. pdf

9. Comisión de Políticas de Enseñanza. Facultad de Ingeniería. Universidad de la República (2002). Estudio de una población recursante. Resol 2628, Exp. $\mathrm{N}^{\circ}$ 06/900-00 II65-04. Version on line: www.fing.edu.uy/uni_ens/Recursantes.pdf

10. Ledesma, I.; Ballestero, P. (2006). Dificultades en el tránsito académico de los alumnos de Primer Año de Psicología. Facultad de Psicología Universidad Nacional de Tucumán. Trabajo 299.E Book Browse. Postgrado Tucumán.

II. Version on line: www.feeye.uncu.edu.ar/.../.tucuman.pdf

12. Fornasero, S.; Martella, L; Tumini, G.(2008). El alumno universitario recursante. Trabajo del Proyecto PIIMEG: Proyecto de innovación e investigación para el mejoramiento de la enseñanza de grado.

13. Lozano, S.; Díaz, A.; Jorrat, I. (2009). Un estudio comparado del rendimiento de estudiantes de Ingeniería frente a una innovación didáctica puesta en práctica en cursos muy masivos. "III Congreso Nacional y II Encuentro Internacional de Estudios Comparados en Educación". SAECE (Soc. Arg. de Estudios Comparados en Educación) Buenos Aires. Universidad de Palermo. Version on line: www.saece.org.ar/docs/congreso3/Ragout.doc

14. Monzón, G.; Di Paolo, J. (2008). El espacio y tiempo oportunos para desarrollar competencias en alumnos ingresantes a ingeniería. Scielo. Ciencia, docencia y tecnología (Entre Ríos). 
15. Version on line: http://www.scielo.org.ar/scielo. php? scrip=sci

16. Blanca, S.; Ávila, M.; Seguir, P.; Menichetti, N.; Flores, D.; Rodriguez, C.; Ferrín, M., Velarde, L. Opinión de los alumnos recursantes sobre su trayectoria en la Universidad. Revista de la Facultad de Medicina. Vol $8-\mathrm{N}^{\circ}$ I: $5 \mathrm{I}-53$.

17. Golbach, M.; Mena, A.; Abraham, G.; Rodríguez, M.; Galindo, G.; Rodriguez Anido, M. (2008). Resultados Académicos conforme a los hábitos y estrategias de aprendizaje. CLAME (Comité latinoamericano de Matemática Educativa). Acta Latinoamericana de Matemática educativa. Vol 2I. México: 255-266. Version on line: www.clame. org.mx/documentos/alme21.pdf

18. Fornasero, S.; Martella, L; Tumini, G. (2006). El alumno universitario recursante. VII Congreso Nacional de Sociología Jurídica.

19. Versión on line: www.planetaius.com.ar > Planeta lus > Eventos > Congresos

20. Medina, C. (2007). El primer año universitario... Estrategias para salir airoso/a en esta etapa.

21. Versión on line: cuhwww.upr.clu.edu/ castula/ primer.pdf

22. Galindo, S.; Cudmani, L. Rendimiento Académico en aulas multitudinarias de Matemática: Evaluación de una estrategia didáctica. Revista Educación y Ciencia. Vol. 8, № 16 (30), pp. 23-36. México.

23. Alderete, M.; Merletti, S.; Pérez, L. (2006) Rendimiento Académico de los aspirantes a ingresar a la Facultad de Odontología de la Universidad Nacional de Tucumán. XXXIX Reunión Anual Sociedad Argentina de Investigación Odontológica. San Miguel de Tucumán.
24. Magnarelli, G.; Quintana, M.; García, L.; Rosso, E.; Cabrera, L.; Ruiz Moreno, L. (2009). El trabajo en pequeños grupos facilita la enseñanza-aprendizaje de Bioquímica. Revista Brasileira de Educacao Médica. Vol 33. N ${ }^{\circ}$ 3:I-II. Versión on line: http:// www.scielo./scielo.php?script=sci

25. D'Angelo, S.; Palacio, S. (2004). Estrategias de integración y rendimiento académico. Universidad Nacional del Nordeste. Comunicaciones Científicas y Tecnológicas. 